\title{
OUTCOMES OF SURGICALLY TREATED NONFUNCTIONING PITUITARY ADENOMAS
}

INTRODUCTION The sequelae of surgically treated non-functioning pituitary adenomas (NFPA) is an important area of study to help plan management. The aim was to study all Maltese patients who had a surgically treated NFPA and analyse the results of surgery, risk factors for tumour recurrence/regrowth and the role of postoperative radiotherapy.

METHODOLOGY 175 patients were identified as having a NFPA of whom 77 had underwent pituitary surgery. Detailed analysis of these patients was done including their demographic details, surgical details, post-surgical management, regrowth and recurrence patterns.

RESULTS $63.6 \%$ of patients presented with visual field defects, $40.3 \%$ had headaches at presentation and $87.0 \%$ had chiasmal compression by their NFPA. Residual tumour postoperatively was evident in $67.5 \%$ of patients while $29.9 \%$ of patients had immediate postoperative radiotherapy. Recurrence /regrowth was documented in $18.2 \%$ of patients within a median time of 3.2 (IQR: 1.6-5.6) years. Factors that were found to be statistically significantly associated with a higher rate of regrowth were the presence of residual tumour $(P=0.036)$, presence of cavernous sinus invasion $(P=0.034)$ and the lack of postoperative radiotherapy ( $P$ $=0.004$ ).

CONCLUSIONS By studying this cohort of patients we were able to characterise better the outcomes of NFPA management and outline risk factors which can effect prognosis.

\section{Gruppetta ${ }^{1,2}$, J. Vassallo,}

Neuroendocrine Clinic, Mater Dei Hospital, Msida, Malta;

${ }^{2}$ Department of Medicine, University of Malta Medical School, Msida, Malta

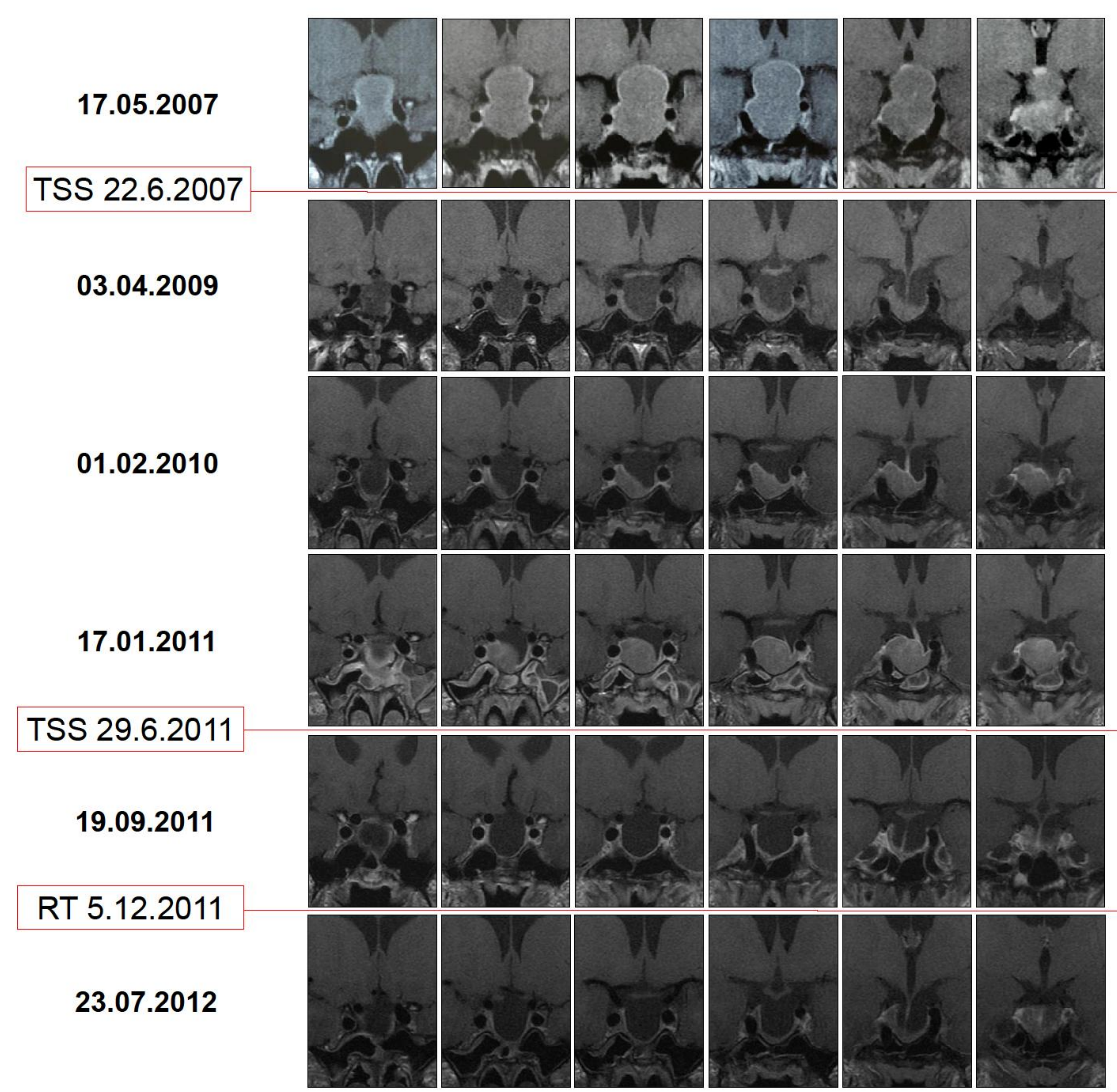

FIGURE 1. Sequential MRI scans of a particular case showing regrowth of residual pituitary adenoma post trans-sphenoidal surgery (TSS), subsequent re-operation and radiotherapy (RT).

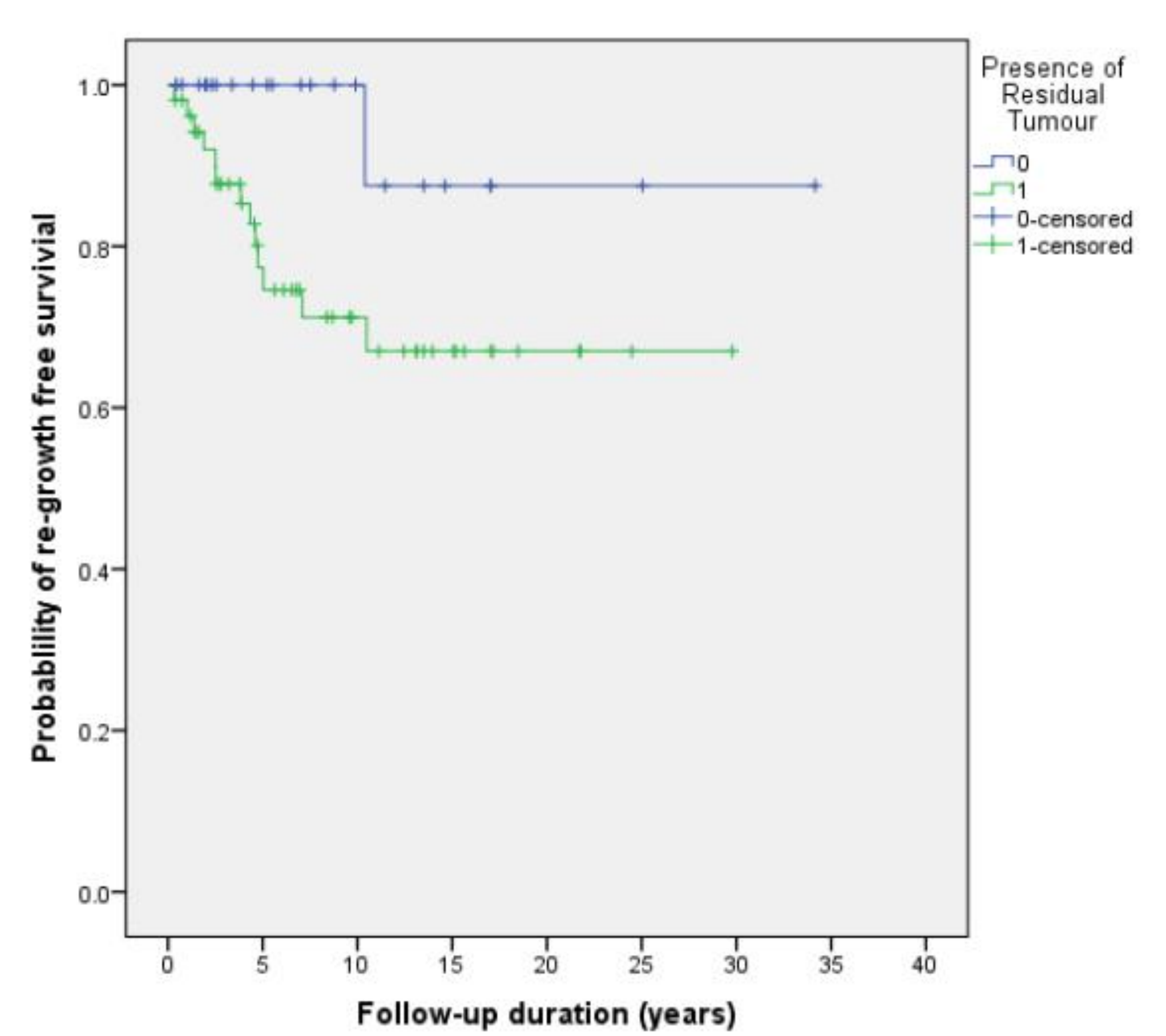

FIGURE 2. Kaplan-Meier estimates of cumulative recurrencefree survival probability classified according to the presence or absence of post-operative residual pituitary adenoma remnant. $(P=0.036)$

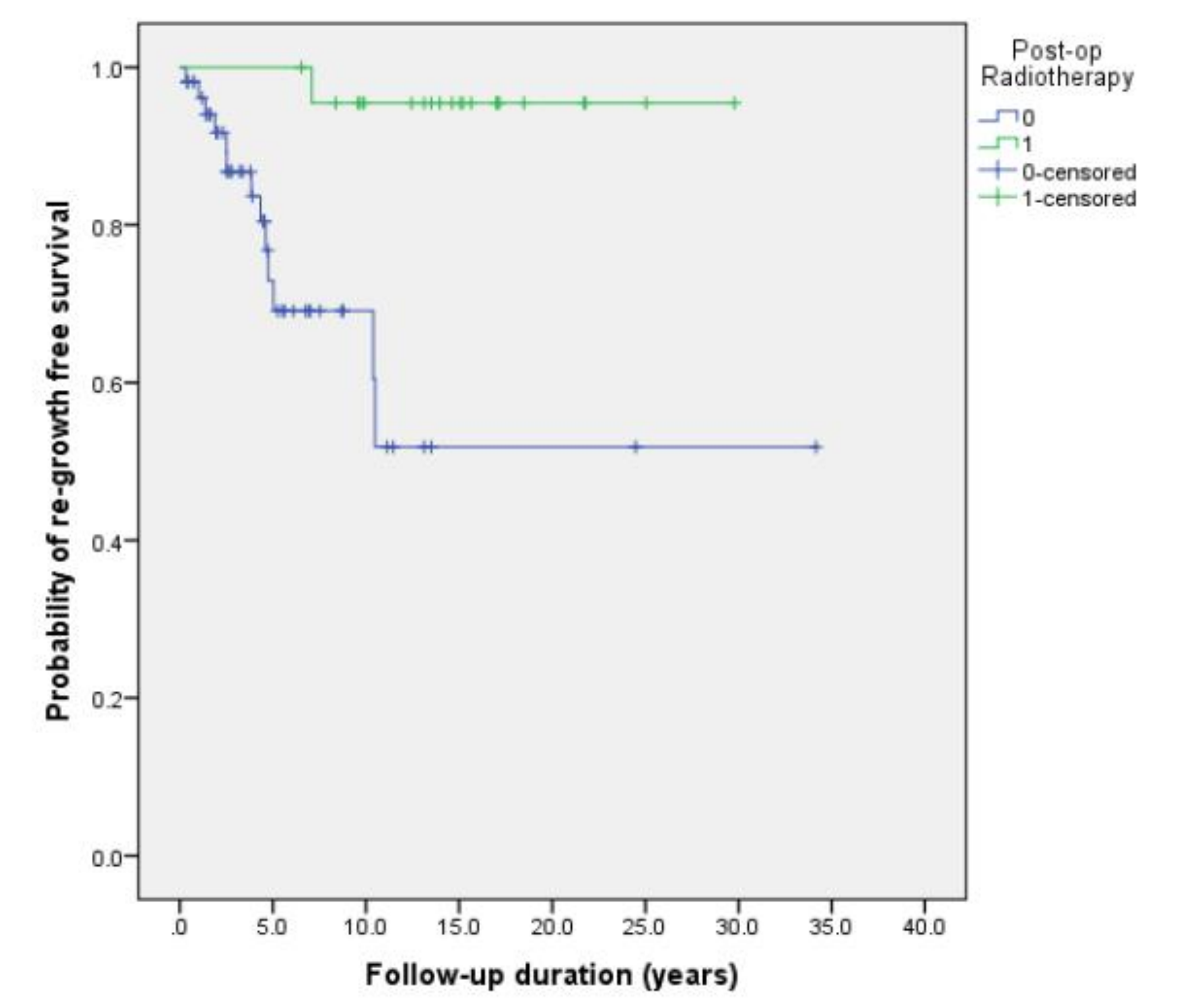

FIGURE 4. Kaplan Meier estimates of cumulative recurrencefree survival probability classified according to whether patients had post-operative radiotherapy. $(P=0.004)$

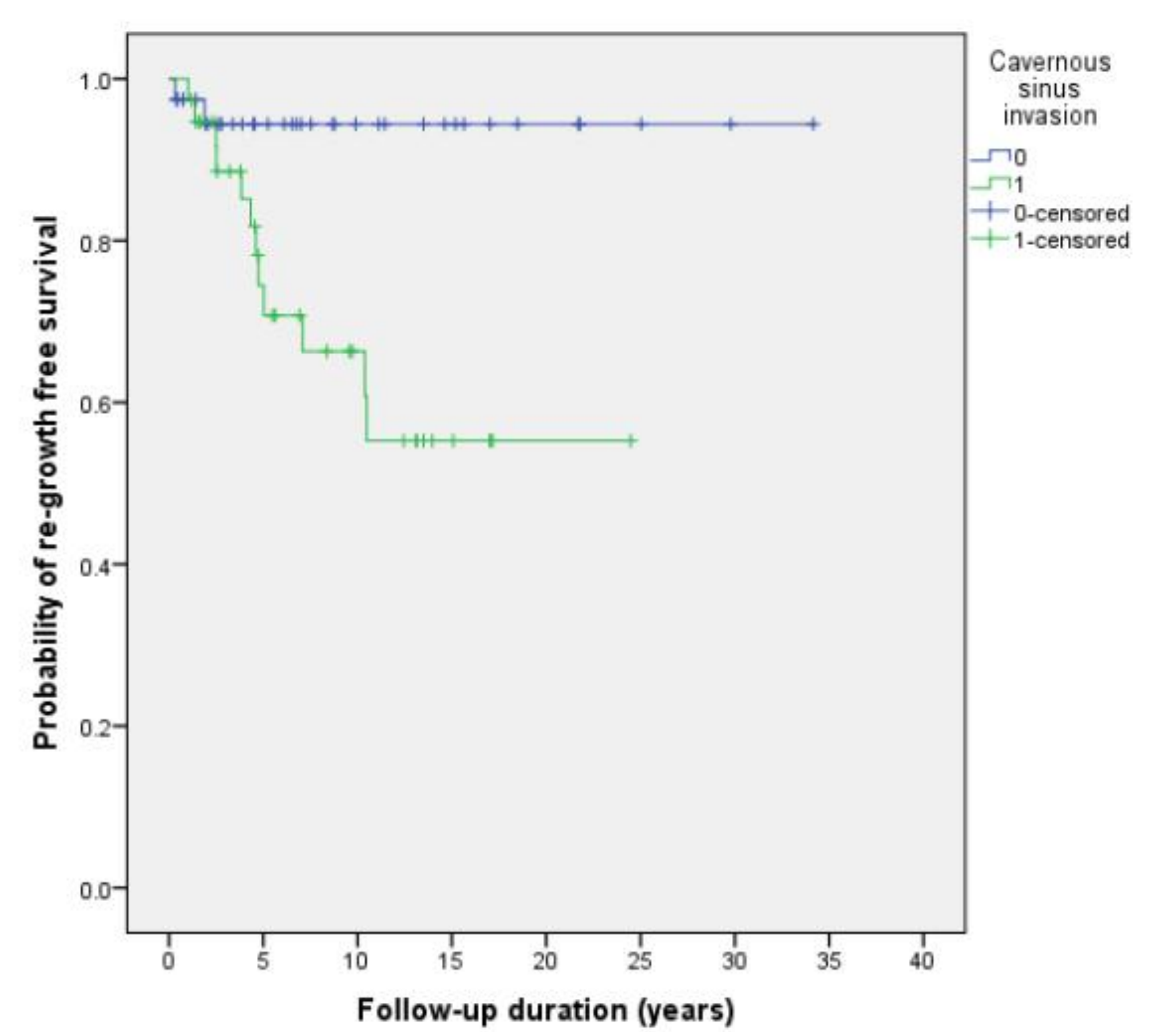

FIGURE 3. Kaplan Meier estimates of cumulative recurrencefree survival probability classified according to whether patients had post-operative radiotherapy. $(P=0.004)$

Independent risk factors for tumour regrowth by multivariate Cox hazard analysis:

-Absence of post-op RT ( $P=0.010)$;

- Cavernous sinus invasion ( $P=0.020)$. 